ISSN: 0213-2087 e-ISSN: 2444-7080

DOI: https://doi.org/10.14201/shhcont372019107115

\title{
FONDOS DOCUMENTALES CONTRA PERSONAL DE LOS ARCHIVOS MILITARES
}

\section{Fonds versus staff at the Military Archives}

\author{
Víctor MORALEDA TORRES \\ Archivero del Estado. Archivo General Militar de Ávila \\ vimorat@gmail.com
}

María del Carmen RIAL QUINTELA

Archivera del Estado. Archivo Intermedio Militar Noroeste

Recibido: 12/07/2019 Revisado: 06/09/2019 Aceptado: 09/09/2019

RESUMEN: Los archivos integrantes del Sistema Archivístico de la Defensa permiten estudiar la Historia de España desde todos los aspectos: militar, político, social, económico y cultural, y de cualquier época, desde el medievo hasta la actualidad. Además, se puede relacionar su documentación con otros centros archivísticos, como los archivos estatales del Ministerio de Educación, Cultura y Deporte. Por tanto, ante la trascendencia de esta documentación, un gran volumen repartido en diversos archivos, habría que pensar si están debidamente dotados de personal y material. En este capítulo vamos a analizar lo que preveía el Reglamento de Archivos Militares y siguiendo los datos proporcionados por el Ministerio de Defensa a través de las estadísticas, comprobaremos el grado de cumplimiento.

Palabras clave: Archivos; gestión documental; defensa; estadísticas.

ABSTRACT: Archives belonging to the Military Archivists System allow to study the Spanish History from every aspect; military, politically, socially, economically and culturally, and from any period, from the Medieval to the present times. These documents can be associated to other Archive Centres, such as the central archives of the Ministry of Education, Culture and Sports. Therefore, with the transcendence of these documents, a big volume is split in different archives. We should be questioning if the Archives are provided with qualified resources and adequate material. In this article, it will be analysed what is the Military Archive Regulation foreseen and examined the degree of compliance using the data provided by the Ministry of Defence through their statistics.

Key words: Archives; document management; defence; statistics. 


\section{COMBATE: FONDOS DOCUMENTALES VERSUS PERSONAL DE LOS ARCHIVOS}

Conocer el estado de situación de los archivos militares requiere analizar una serie de información y datos para poder saber cuál era la situación de partida y cuál es la actual, y así poder trazar correctamente su evolución. Para ello, en este combate, hemos acudido a las Estadísticas de centros, instalaciones y actividades culturales y deportivas del Ministerio de Defensa. Hemos empezado en el año 2000 por dos motivos: el primero, por ser un año simbólico al cambiar de siglo, y; el segundo, porque el Reglamento de Archivos Militares (en adelante, RAM) llevaba ya un año aprobado y, por tanto, dicho año supone el inicio de una nueva era en los archivos militares.

De las estadísticas, nos hemos centrado en los datos específicos de volumen documental, de personal (tanto militar como civil) y, a partir del año 2009, también del personal técnico (facultativos, ayudantes y auxiliares) destinado en los archivos. También hay que tener presente que entre 2000 y 2008 los datos hacen referencia única y exclusivamente a archivos históricos. A pesar de que los antiguos archivos regionales (que a raíz del Real Decreto 2598/1998 se transformaron en archivos intermedios) se crean en el año 1995, por Norma General 5/95, no se publican sus estadísticas hasta el año 2009. Lo mismo ocurre con el archivo del Órgano Central, del que hasta el año 2011 no se empieza a conocer información.

De esta forma, y con está casuística, podemos ver en la tabla siguiente la evolución en el volumen documental que se custodia y el personal encargado de su tratamiento y puesta a disposición de la sociedad, que es la verdadera destinataria de la tarea que se realizan en los centros.

\subsection{Años 2000-2008: sólo archivos históricos}

Tabla 1. Volumen y personal de los archivos históricos militares entre 2000 y 2008. Fuente:

Estadísticas anuales de los archivos militares publicadas por el Ministerio de Defensa

\begin{tabular}{|l|c|c|c|c|c|c|c|c|c|}
\hline SAD & 2000 & 2001 & 2002 & 2003 & 2004 & 2005 & 2006 & 2007 & 2008 \\
\hline Volumen $^{1}$ & 56.619 & 57.970 & 57.609 & 58.358 & 57.321 & 58.173 & 58.622 & 58.909 & 59.957 \\
\hline Personal & 140 & 137 & 157 & 154 & 148 & 156 & 154 & 165 & 150 \\
\hline
\end{tabular}

En el año 2000, un año después de ser publicado el Reglamento, el volumen de los fondos integrantes de los tres Subsistemas Archivísticos de la Defensa, es decir, Ejército de Tierra, Marina y Ejército del Aire, en sus archivos históricos, ocupa 56.619 metros lineales. Respecto al personal encargado de gestionar dicho volumen, había 140 personas entre personal militar y civil (no hay datos de cuántos de ellos eran técnicos

1. Se mide en metros lineales: se trata de la longitud de los estantes, que corresponde a un metro de largo y unos 26 centímetros de ancho. 
de archivos). Teniendo en cuenta que son diez los archivos históricos ${ }^{2}$ los que estamos tratando, ello supondría una media de 14 personas por centro, pero analizando detenidamente los datos de la Estadística de centros del Ministerio de Defensa se comprueba que no es así. Existen unas diferencias muy llamativas en los distintos Subsistemas: en la Armada hay una escasez total; en el Ejército del Aire puede considerarse que se mantuvo suficientemente dotado de personal, y; en el Ejército de Tierra, hay diversidad de unos centros a otros. Hemos de presuponer que el personal técnico de archivos debía ser escaso. El Jefe de la Unidad de Coordinación de Archivos del Ministerio de Defensa, Antonio González Quintana, promotor de la redacción del Reglamento de Archivos Militares, establecía en 50 técnicos el personal idóneo para gestionar los fondos integrantes del Sistema de Archivístico de la Defensa.

El volumen documental en este periodo de tiempo pasa de 56.619 a 59.957 metros lineales, se produce un cierto ascenso de 3.338 metros lineales. En los años 2002 y 2004, por razones que se desconocen, se producen una serie de descensos, para luego ascender. Esta anomalía se produce en el Subsistema archivístico del Ejército de Tierra, como se puede apreciar en la siguiente tabla:

Tabla 2. Volumen de fondos documentales de los archivos históricos del Ejército de Tierra entre 2000 y 2008. Fuente: Estadísticas anuales de los archivos militares publicadas por el Ministerio de Defensa

\begin{tabular}{|l|c|c|c|c|c|c|c|c|c|}
\hline E. Tierra & 2000 & 2001 & 2002 & 2003 & 2004 & 2005 & 2006 & 2007 & 2008 \\
\hline Volumen & 40.163 & 41.329 & 40.577 & 40.771 & 39.448 & 40.102 & 40.443 & 40.566 & 41.526 \\
\hline
\end{tabular}

Respecto al personal, la evolución es mucho más irregular. En el año 2000 se cuenta con 140 personas, dos años después repunta a 157, en los años 2003 y 2004 desciende hasta 154 y 148, en el siguiente par de años asciende para llegar al 2007 a su cota máxima de personal de este periodo de tiempo que estamos analizando, con 165 personas destinadas en los archivos, entre militares y civiles, para llegar al 2008 con un nuevo descenso, 150.

Entre los Subsistemas, vuelve a ser el Ejército de Tierra el que mayor fluctuación de personal presenta, al partir en el año 2000 con 105 personas, para llegar a su techo en los años 2002, 2003 y 2007 y terminar este ciclo como había empezado con 104, el segundo peor año después del 2001 en el que hubo solo 101 personas destinadas

2. Se tratan de: Ejército de Tierra: Archivo General Militar de Madrid, Archivo General Militar de Segovia, Archivo General Militar de Guadalajara, Archivo General Militar de Ávila y Cartoteca Histórica del Servicio Geográfico del Ejército; Armada: Archivo del Museo Naval, Archivo General de Marina Álvaro de Bazán, Archivo histórico del Real Instituto y Observatorio de la Armada y Servicio Histórico del Instituto Hidrográfico de la Marina, y; Ejército del Aire: Archivo General e histórico del Aire. 
en los archivos del Ministerio de Defensa. En comparación, los centros de la Armada contaron con un personal más estable, 9-10 personas. Pero fue el Ejército del Aire quien contó con una política de personal más efectiva para los archivos ya que incrementó su personal anualmente, pasando de 25 personas en el año 2000 a 37 al final del ciclo.

Tabla 3. Personal de los archivos históricos militares entre 2000 y 2008, por ejércitos. Fuente:

Estadísticas anuales de los archivos militares publicadas por el Ministerio de Defensa

\begin{tabular}{|l|c|c|c|c|c|c|c|c|c|}
\hline Personal & 2000 & 2001 & 2002 & $\mathbf{2 0 0 3}$ & $\mathbf{2 0 0 4}$ & $\mathbf{2 0 0 5}$ & $\mathbf{2 0 0 6}$ & $\mathbf{2 0 0 7}$ & $\mathbf{2 0 0 8}$ \\
\hline E. Tierra & 105 & 1001 & 118 & 116 & 110 & 109 & 108 & 118 & 104 \\
\hline Armada & 10 & 10 & 9 & 9 & 9 & 9 & 10 & 10 & 9 \\
\hline E. Aire & 25 & 26 & 30 & 29 & 29 & 38 & 36 & 37 & 37 \\
\hline
\end{tabular}

\subsection{Años 2009-2017: la irrupción de los archivos intermedios}

Tabla 4. Volumen y personal de los archivos militares entre 2009 y 2017. Fuente: Estadísticas anuales de los archivos militares publicadas por el Ministerio de Defensa

\begin{tabular}{|l|c|c|c|c|c|c|c|c|c|}
\hline & 2009 & 2010 & 2011 & 2012 & 2013 & 2014 & 2015 & 2016 & 2017 \\
\hline Volumen & 131.013 & 128.626 & 150.429 & 152.441 & 151.540 & 149.617 & 148.138 & 151.600 & 155.997 \\
\hline Personal & 264 & 302 & 320 & 354 & 325 & 316 & 312 & 284 & 274 \\
\hline Técnicos & 21 & 29 & 36 & 26 & 27 & 27 & 29 & 18 & 21 \\
\hline
\end{tabular}

Gráfico 1. Incremento del volumen de fondos documentales de los archivos militares entre 2000 y 2017, empezándose a contabilizar los fondos de los archivos intermedios a partir de 2009. Fuente: Estadísticas anuales de los archivos militares publicadas por el Ministerio de Defensa

Ejército de Tierra, Archivos Histórico y Archivos Intermedios

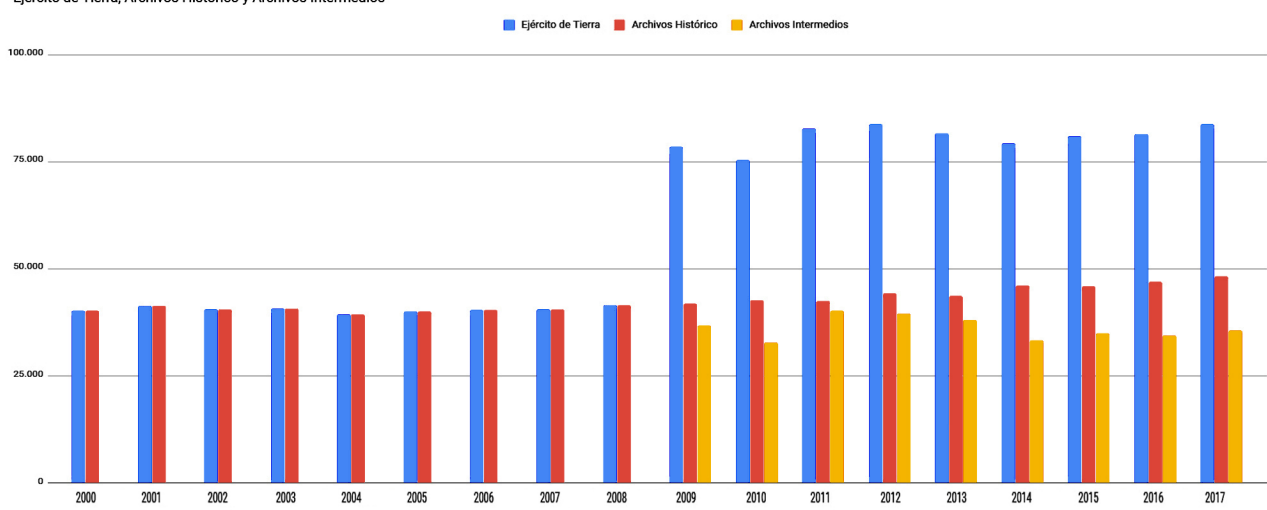


Gráfico 2. Incremento del volumen de fondos documentales de los archivos militares entre 2000 y 2017, y evolución del personal destinado, y personal técnico diferenciado a partir de 2009. Fuente: Estadísticas anuales de los archivos militares publicadas por el Ministerio de Defensa.

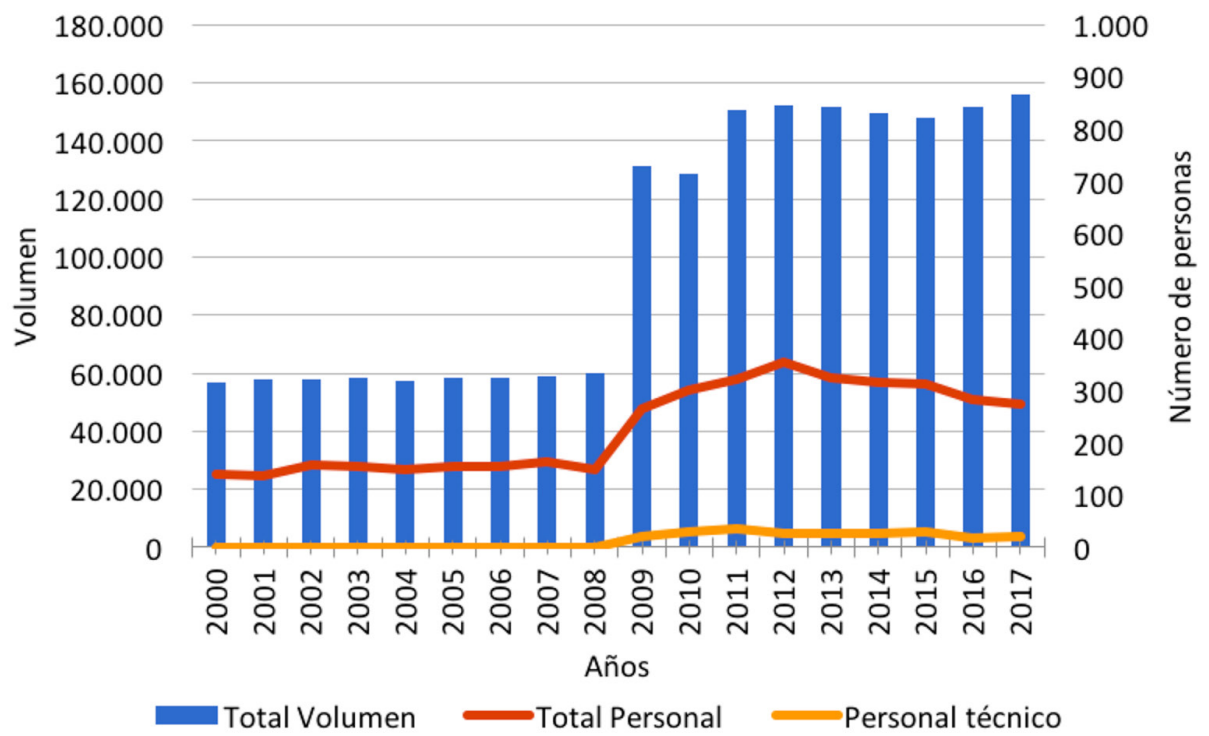

El inicio de este nuevo ciclo a analizar también tiene una doble motivación, ya que en 2008: uno, llevaba un año aprobada la Ley 52/2007, de 26 de diciembre, por la que se reconocen y amplían derechos y se establecen medidas a favor de quienes padecieron persecución o violencia durante la guerra civil y la dictadura o de la Memoria Histórica, y; dos, las Estadísticas de centros del Ministerio de Defensa empezaron a publicar los datos de los archivos intermedios ${ }^{3}$. Si en el ciclo anterior, tratábamos datos sobre catorce centros, en esta nueva fase, son 26 los centros que proporcionan información estadística.

El Sistema Archivístico de la Defensa empieza el ciclo con 131.013 metros lineales de documentación para llegar al 2017 con 155.997. Al igual que en el ciclo anterior, en

3. Se tratan de: Ejército de Tierra: Archivo General del Cuartel General del Ejército, Archivo Intermedio Militar de Baleares, Archivo Intermedio Militar Centro, Archivo Intermedio Militar de Canarias, Archivo Intermedio Militar de Ceuta, Archivo Intermedio Militar de Melilla, Archivo Intermedio Militar Noroeste, Archivo Intermedio Militar Pirenaico y Archivo Intermedio Militar Sur; Armada: Archivo Central del Cuartel General de la Armada, Archivo Naval de Canarias, Archivo Intermedio de Cartagena, Archivo Intermedio de Ferrol y Archivo Naval de San Fernando, y; Ejército del Aire: Archivo General del Cuartel General del Ejército del Aire. Además, se incluye el Archivo General e Histórico de Defensa, integrante del Órgano Central del Ministerio de Defensa. 
los años 2010, 2013, 2014 y 2015 se reflejan unos descensos en el volumen documental y vuelve a ser el Ejército de Tierra el Subsistema que presenta dicha anomalía. También hay que resaltar el hecho que a partir del año 2011 se publican las estadísticas del Órgano Central, referente al Archivo General e Histórico de Defensa, pero resalta una particularidad: entre 2011 y 2014 refleja 9.000 metros lineales de documentación pero en 2015 baja a 5.314, seguir subiendo al año siguiente hasta casi los ocho mil metros lineales y llegar al 2017 a los 18.900 metros lineales, es decir, duplicando el volumen de documentación que tenía el año anterior.

En el apartado de personal, vamos a distinguir, por un lado, al personal total (militares y civiles) y, por otro lado, al personal técnico en los archivos militares. Así, arrancamos el ciclo con 264 personas, incrementándose anualmente hasta llegar a la cumbre del ciclo en el 2012, en el que los archivos contarán con un total de 354 personas ligadas a ellos. A partir de aquí, se va produciendo un declive constante e imparable, llegando al 2017 (último año en el que tenemos datos) con 274 personas destinadas en los archivos. Es decir, el ciclo se termina prácticamente en la misma situación con la que empezó respecto al personal, pero con una diferencia mayúscula: el volumen documental ha crecido en casi 25 kilómetros lineales de documentación.

Si analizamos los datos según los distintos subsistemas podemos ver que el Ejército de Tierra fue aumentando su personal ligeramente, llegando a su cénit en 2014, con 201 personas destinadas en los archivos, pero a partir de ahí se ha producido un descenso muy marcado, sobre todo en los años 2016 y 2017 llegando a tocar fondo, con 179 y 171 personas. La Armada es un caso significativo porque empieza con 34 personas y al año siguiente, prácticamente, duplica su personal llegando a su cumbre en el 2012 con 102 personas para, al año, volver a la situación previa y mantenerse estable, pero en descenso paulatino. El Ejército del Aire, sin embargo, es un Subsistema bastante estable en cuanto a personal ya que durante todo el ciclo su personal se encuentra entre las 42 y 45 personas produciéndose un descenso en el año 2017, bajando a las 36 personas. El órgano central, al igual que el Aire, se mantiene en un cierto equilibrio de personal.

Tabla 5. Personal de los archivos militares entre 2008 y 2017, por subsistema. Fuente: Estadísticas anuales de los archivos militares publicadas por el Ministerio de Defensa

\begin{tabular}{|l|c|c|c|c|c|c|c|c|c|}
\hline Personal & $\mathbf{2 0 0 9}$ & $\mathbf{2 0 1 0}$ & $\mathbf{2 0 1 1}$ & $\mathbf{2 0 1 2}$ & $\mathbf{2 0 1 3}$ & $\mathbf{2 0 1 4}$ & $\mathbf{2 0 1 5}$ & $\mathbf{2 0 1 6}$ & $\mathbf{2 0 1 7}$ \\
\hline E. Tierra & 186 & 197 & 199 & 198 & 199 & 201 & 194 & 179 & 171 \\
\hline Armada & 34 & 63 & 66 & 102 & 69 & 62 & 65 & 56 & 58 \\
\hline E. Aire & 44 & 42 & 44 & 44 & 45 & 44 & 45 & 42 & 36 \\
\hline Ó. Central & 0 & 0 & 4 & 2 & 4 & 4 & 4 & 2 & 3 \\
\hline
\end{tabular}

Respecto al personal técnico, el ciclo empieza y termina con la misma cantidad de personas adscritas, es decir, con 21. Como hemos visto anteriormente, su momento 
de mayor esplendor es en el 2011, con 36 técnicos de archivos para ir descendiendo tímidamente hasta que en el 2016 se refleja una bajada brusca, llegando únicamente a los 18 archiveros, remontando levemente al año siguiente.

Comparando los distintos Subsistemas, la Armada ha sido la más desfavorecida de todas ya que siendo sus momentos dorados los años 2010 y 2011 con 11 y 13 técnicos, respectivamente, finaliza el ciclo de forma pobre, al contar con 1 y 3 en los años 2016 y 2017. Mientras que el Ejército de Tierra y el Ejército del Aire, junto al Órgano Central podemos decir que mantiene una estabilidad de personal, pero siempre escaso.

Tabla 6. Personal técnico de archivos de los archivos militares entre 2009 y 2017, por subsistema.

Fuente: Estadísticas anuales de los archivos militares publicadas por el Ministerio de Defensa

\begin{tabular}{|l|c|c|c|c|c|c|c|c|c|}
\hline Técnicos & $\mathbf{2 0 0 9}$ & $\mathbf{2 0 1 0}$ & $\mathbf{2 0 1 1}$ & $\mathbf{2 0 1 2}$ & $\mathbf{2 0 1 3}$ & $\mathbf{2 0 1 4}$ & $\mathbf{2 0 1 5}$ & $\mathbf{2 0 1 6}$ & $\mathbf{2 0 1 7}$ \\
\hline E. Tierra & 10 & 14 & 16 & 14 & 14 & 16 & 12 & 12 & 13 \\
\hline Armada & 7 & 11 & 13 & 8 & 7 & 5 & 8 & 1 & 3 \\
\hline E. Aire & 4 & 4 & 3 & 2 & 2 & 2 & 5 & 3 & 2 \\
\hline Ó. Central & 0 & 0 & 4 & 2 & 4 & 4 & 4 & 2 & 3 \\
\hline
\end{tabular}

\subsection{Años 2018-2019: presente de los archivos militares}

El 16 abril de 2016 empezaron las oposiciones y el 13 de febrero de 2017 empezaron el curso práctico los facultativos y ayudantes de archivos que habían aprobado, el cual se da por finalizado a finales de mayo. Es a finales de septiembre cuando los nuevos archiveros del Estado toman posesión de sus puestos. Debido a este retraso en la convocatoria de 2015, en el 2018 no hubo ninguna incorporación de nuevos técnicos de archivos en los archivos estatales; estos se producen en febrero de 2019, procedentes de la convocatoria de 2016. Actualmente, se encuentra en proceso la convocatoria de 2017.

En el 2018, se produjeron, a falta de que se publique la estadística anual, dos bajas de personal, las técnicas del Archivo Central del Ministerio de Defensa y del Archivo Intermedio Militar Centro (que se encuentra en Valencia), provocando que ambos centros se queden sin personal técnico.

Sin embargo, en febrero de 2019, procedentes de la última convocatoria de oposiciones a los Cuerpos de Facultativos y Ayudantes de Archivo, se han incorporado 17 técnicos (10 facultativos y 7 ayudantes) al Sistema Archivístico de la Defensa. Pero la dicha no es completa, porque la mayor parte de ellos no han ido a los archivos que son los que pueden responder las consultas de los ciudadanos con los documentos que custodian, abordar las valoraciones y transferencias previstas por la normativa, o, en el caso de los archivos intermedios, coordinar y apoyar a los cientos de archivos centrales de Regimientos, buques y otras unidades militares que igualmente custodian patrimonio documental militar. 


\section{CONCLUSIONES}

Antes de centrar las conclusiones objeto de este trabajo relativas a personal de los archivos militares, queremos mencionar la escasez de medios materiales en los centros y, mayoritariamente, en muchos casos obsoletos tecnológicamente. El caso más llamativo es el hecho de que sólo cuatro archivos (históricos) cuentan con el programa de gestión documental ArchiDoc (software desfasado y sin mantenimiento por parte de la empresa creadora, El Corte Inglés, S.A.). Así, se sigue esperando que desde el Ministerio de Defensa se decidan por implantar un nuevo software que abarque a todo el SAD o integrarse dentro de la plataforma PARES 2.0 del Ministerio de Cultura, solución más económica, rápida y sencilla (no debemos olvidar que una gran parte del escaso personal técnico en los archivos militares procede del Ministerio de Cultura y con amplia experiencia en el trabajo en dicho gestor documental). Así, todos los centros tendrían un software común normalizado y no existirían las diferencias como hemos constatado, y las personas podrían buscar los documentos de su interés al mismo tiempo en los inventarios de los archivos de ambos ministerios.

Respecto a personal, el Título III del RAM establece la plantilla básica con la que debieran estar dotados los centros, tanto personal técnico, como auxiliar y administrativo. Si nos atenemos a los datos, podemos comprobar que no se cumple el Reglamento ya que hay centros que carecen de personal técnico, otros de personal auxiliar y en otros no hay personal ni de los primeros ni de los segundos. Estas carencias provocan varias incidencias todos los años:

En primer lugar, las cifras de personal que se han expuesto son insuficientes para prestar los servicios de los archivos militares, como prueba el hecho de que ha habido ocasiones en que se llegado a cerrar oficialmente algún centro (el Archivo General de Marina Álvaro de Bazán estuvo varios meses cerrado a los investigadores debido a que la dirección técnica quedó vacante y no había más personal técnico que pudiera asumirla interinamente) y en otras ocasiones se ponen condiciones de acceso a las salas de investigadores (el Archivo General Militar de Segovia y el Archivo General Militar de Ávila tienen sufren carencia de personal subalterno que supone que haya que pedir cita previa para la consulta de documentación de forma presencial).

En segundo lugar, llamamos la atención sobre el hecho de que estas limitaciones de servicio archivístico con que se encuentra la ciudadanía a priori son evidentes, ya que se manifiestan en no contestaciones a consultas, cierres de Salas de Investigación, no tramitación de peticiones de reproducción, etc. Pero no son menos importantes las limitaciones de calidad de los servicios, que saltan menos a la vista, como las relativas a que estas carencias de técnicos y personal auxiliar en los centros obliga a externalizar trabajos archivísticos tales como descripción de series documentales, digitalización de documentación o el servicio de ordenanzas. Esto agrava el problema porque en muchas ocasiones sucede que no se logra hacer un seguimiento y control de los trabajos.

En tercer lugar, hay consecuencias en el servicio de referencias y atención a la investigación, ya que la falta de personal técnico hace habituales las respuestas que se realizan sin intervención de personal archivero, bien porque no se cuenta con ninguna 
plaza cubierta, bien porque no alcanza a revisar todas las peticiones de antecedentes, bien porque se prescinde de su criterio y experiencia. Dado que cualquier consulta o petición de antecedentes es firmada por el director militar del archivo del Ministerio de Defensa de que se trate, la persona que la presenta desconoce si un profesional de la archivística ha intervenido en la búsqueda documental y en la redacción de la respuesta que recibe, o si carece de dicha intervención por lo cual puede ser una respuesta de calidad deficiente, y por supuesto carecerá de cualquier componente científico. Las deficiencias de calidad en el servicio de referencias que pueden producirse cuando no interviene un archivero son variadas: la búsqueda no ha sido adecuada, la redacción de la respuesta no aporta el contexto necesario para comprender el documento, se deniega el acceso cuando corresponde facilitarlo, se facilitan copias de documentos a los que afecta algún tipo de restricción,...

En cuarto lugar, los datos estadísticos publicados por el Ministerio de Defensa demuestran que un incremento de personal implica un aumento de los servicios que se prestan a la sociedad. El salto cuantitativo que se produce entre finales de 2009 (264 personas en los archivos militares históricos e intermedios) y finales de 2012 (354 personas) va acompañado de un incremento constante de las consultas presenciales y no presenciales que se contestan en los archivos militares, mientras que la pérdida de personal a partir de ese 2012, especialmente de personal técnico de archivos, se hace notar igualmente en las consultas de los años siguientes, como puede comprobarse en la siguiente tabla:

Tabla 7. Consultas presenciales y no presenciales de los archivos militares entre 2009 y 2017 , personal total y personal técnico. Fuente: Estadísticas anuales de los archivos militares publicadas por el Ministerio de Defensa

\begin{tabular}{|l|c|c|c|c|c|c|c|c|c|}
\hline SAD & 2009 & 2010 & 2011 & $\mathbf{2 0 1 2}$ & $\mathbf{2 0 1 3}$ & $\mathbf{2 0 1 4}$ & $\mathbf{2 0 1 5}$ & $\mathbf{2 0 1 6}$ & $\mathbf{2 0 1 7}$ \\
\hline Sala & 42.738 & 56.015 & 66.541 & 93.007 & 52.062 & 35.152 & 34.887 & 36.114 & 32.494 \\
\hline Correo & 12.515 & 30.783 & 29.878 & 29.307 & 7.685 & 13.718 & 13.949 & 9.089 & 13.365 \\
\hline Personal & 264 & 302 & 320 & 354 & 325 & 316 & 312 & 284 & 274 \\
\hline Técnicos & 21 & 29 & 36 & 26 & 27 & 27 & 29 & 18 & 21 \\
\hline
\end{tabular}

Como conclusión global, insistimos en que desde la profesión y también desde los colectivos interesados en los archivos militares, se siga reivindicando el hecho de que ningún archivo esté sin archivero porque va en contra de la ley de patrimonio al dejar indefenso un vasto campo documental sin personal técnico que lo custodie, lo conserve, lo organice y lo difunda.

Todo lo anterior, es un toque de atención para poder llevar a los archivos militares a una situación de control y visualización para un mayor conocimiento de la sociedad. 